\title{
Physical modeling of stresses caused by volume centrifugal forces in a compound rotation body
}

\author{
Elephan Agakhanov ${ }^{1}$, Murad Agakhanov ${ }^{2, *}$ \\ ${ }^{1}$ Dagestan State Engineering University, Imam Shamil Avenue, 70, 367015, city of Makhachkala, \\ Russia \\ ${ }^{2}$ Moscow state university of civil engineering, Yaroslavskoye shosse, 26, Moscow, Russia, 129337
}

\begin{abstract}
The article considers the question of physical modeling of stresses in a compound rotation body of a complex form with a complex loading distribution. According to the similarity parameters, the stresses, deformations and displacements caused by the volume forces are reduced proportionally to the similarity scale for geometrical dimensions which complicates their direct modeling with the use of the models made from conventional epoxy materials. The general methods of study of stresses and deformations usually register them with an inadequate sensitivity. On the basis of the independence principle, the initial problem is presented as a superposition of two problems. In the first (uniform) problem, the stresses in a rotation body caused by centrifugal forces are simulated through the standard "freezing" method. For the solution of the second (non-uniform) problem, the "freezing" of stresses is carried out in the model domain corresponding to the centrifugal forces acting there, and, at the room temperature, the models in their natural state are glued to the model, and the "annealing" of the compound model is performed. The components of radial, tangential and axial stresses at the outlines as well as the cross-sections of models have been obtained through the methods of normal translucence and of numerical integration of the equilibrium equation.
\end{abstract}

\section{Introduction}

The modeling of the problems in mechanics of deformable solid bodies is carried out with the help of the similarity parameters, on the basis of which the model is performed, the loading conditions are determined, and the transition from the measured model values to the corresponding values for the full-scale structure is put into practice. The similarity factors for stresses $K_{\sigma}(t)$, deformations $K_{\varepsilon}(t)$, displacements $K_{u}(t)$, geometrical dimensions $K_{L}$, volume forces $K_{F}(t)$ and elasticity modulus $K_{E}$ in the process of modeling of problems in the creep theory and the elasticity theory (in the problems of the elasticity, the similarity factors become constant values, i.e. similarity scales) are connected through the following formules [1].

\footnotetext{
${ }^{*}$ Corresponding author: muradak@mail.ru
} 


$$
\frac{K_{L} K_{F}(t)}{K_{\sigma}(t)}=1 \frac{K_{L} K_{F}(t)}{K_{\varepsilon}(t) K_{E}}=1 \quad \frac{K_{L}^{2} K_{F}(t)}{K_{u}(t) K_{E}}=1 .
$$

It follows from (1) that the stresses, deformations and displacements caused by volume forces are reduced proportionally to the scale of similarity of geometrical dimensions. This phenomenon complicates a direct modeling even for the most simple case of volume forces (mass forces) through the photo-elasticity method with the use of models made from conventional epoxy materials. The general methods of study of stresses and deformations usually register them with an inadequate sensitivity. The modeling procedures for volume forces are considered in works [2, 3, 4].

When modeling the problems with different characteristics $v, E, \gamma, \alpha$ in different body domains (in contrast to the problems when these characteristics have constant values in the whole body), we have to observe some additional relationships between the similarity factors (scales) [5]:

$$
\begin{gathered}
K_{v 1}=K_{v 2}=\ldots=K_{v n}=1, \\
K_{E 1}=K_{E 2}=\ldots=K_{E n}, \\
K_{F 1}(t)=K_{F 2}(t)=\ldots=K_{F n}(t), \\
K_{\alpha \Delta T}=1 K_{\alpha 1}=K_{\alpha 2}=\ldots=K_{\alpha n},
\end{gathered}
$$

where $\mathrm{n}$ is the number of domains with different characteristics.

These conditions create some restrictions and difficulties for the use of traditional techniques. Some new potentialities for the solution of such problems may appear with the use of the properties of polymers displayed during the process of polymerization (methods of polymerization, straitened shrinkage and fixation of temperature stresses) [6].

In addition to that, the methods are being developed especially for the determination of stresses caused by mechanical loading and temperature on the models made from a standard optical sensitive material in the problems with different characteristics in different body domains [7].

Taking into consideration the aforesaid methods allowing us to obtain effective solutions, we take the characteristics $v, E, \alpha$ to be constant in the whole body in the process of solution of the problem of effect of volume forces.

\section{Methodology}

The engineering practice, in particular, the machine building, more and more uses compound (composite) structural elements made from materials with different volume masses. In their earlier works, the authors considered only uniform rotation bodies [8]. The analysis of stress-and-strain state of compound structural elements is reduced to the solution of complicated systems of partial differential equations, the direct solution of which is quite difficult. Thus, many important practical problems for compound bodies cannot be solved theoretically yet. The determination of stress-and-strain state of compound structural elements with complicated forms and with a complicated distribution of loading is one of such problems. 
Let us consider a volume rotation body composed of two materials with the density values $\rho_{1}$ and $\rho_{2}$ (Fig. 1). This compound structural element rotates with the angular velocity $\omega_{f s c}$, and it is under the influence of volume centrifugal forces

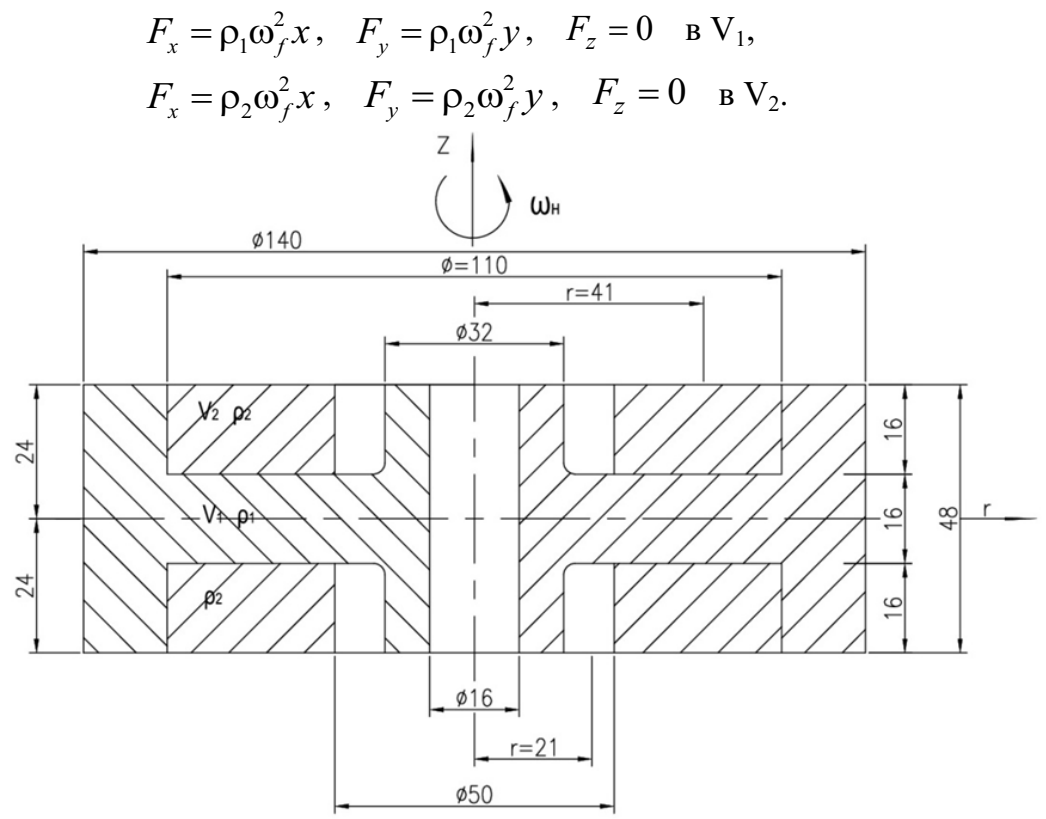

Fig. 1. Compound rotation body under the effect of centrifugal forces.

\section{Main part}

We use the principle of independence of action of forces and interpret the solution of the initial problem as the sum of two problems.

1. In the whole compound body $\left(\mathrm{V}=\mathrm{V}_{1}+\mathrm{V}_{2}\right)$ the following volume forces act:

$$
F_{x}=\rho_{2} \omega_{f s c}^{2} x, \quad F_{y}=\rho_{2} \omega_{f s c}^{2} y, \quad F_{z}=0 \quad \text { в V. }
$$

2. The compound body is under the action of the volume forces

$$
\begin{gathered}
F_{x}=\left(\rho_{1}-\rho_{2}\right) \omega_{f s c}^{2} x, F_{y}=\left(\rho_{1}-\rho_{2}\right) \omega_{f s c}^{2} y, \mathrm{~F}_{\mathrm{z}}=0 \quad \text { в } \mathrm{V}_{1}, \\
\mathrm{~F}_{\mathrm{x}}=\mathrm{F}_{\mathrm{y}}=\mathrm{F}_{\mathrm{z}}=0 \text { в } \mathrm{V}_{2} .
\end{gathered}
$$

In the process of modeling of these problems, a standard optical sensitive material is used with the following characteristics

$$
T_{\mathrm{fr}}=130^{0} \mathrm{C}, \quad E_{\mathrm{fr}}=20,0 \mathrm{MPa}, \quad v_{f r}=0.5, \quad \sigma_{0, f r}^{(1,0)}=0,046 \mathrm{MPa} .
$$

The stresses in a uniform rotation body caused by centrifugal forces in the first problem are simulated as usual: in the model of the $\mathrm{V}$ domain with the angular rotation velocity $\omega_{\text {mod }}$, the "freezing" of stresses corresponding to the unknown quantities is carried out.

For the solution of the second problem, we perform in the model of the $\mathrm{V}_{1}$ domain the "freezing" of stresses corresponding to the centrifugal forces acting in the $\mathrm{V}_{1}$ domain. Further, we glue to the model of the $\mathrm{V}_{1}$ domain at room temperature the models of the $\mathrm{V}_{2}$ domain which are in their natural state, and then we carry out the "annealing" of the composed model. 
The methods of normal translucence of sections and of numerical integration of the equilibrium equation along the z-axis gave the components of radial $\sigma_{r}$, tangential $\sigma_{\theta}$ and axial $\sigma_{z}$ stresses at the outlines of the models and at two cross-sections.

The stress diagrams in the models are shown in Figures 2-4.

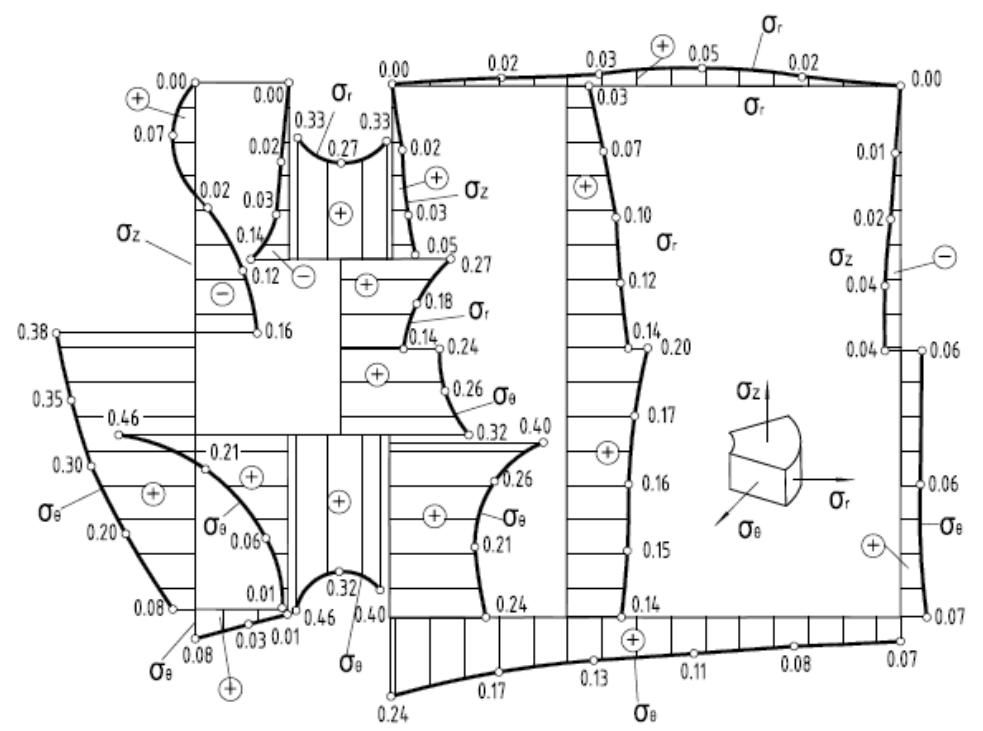

Fig. 2. Stress diagrams $\sigma_{r}, \sigma_{\theta}$ и $\sigma_{z}[\mathrm{MPa}]$ in the model of the $\mathrm{V}$ domain.

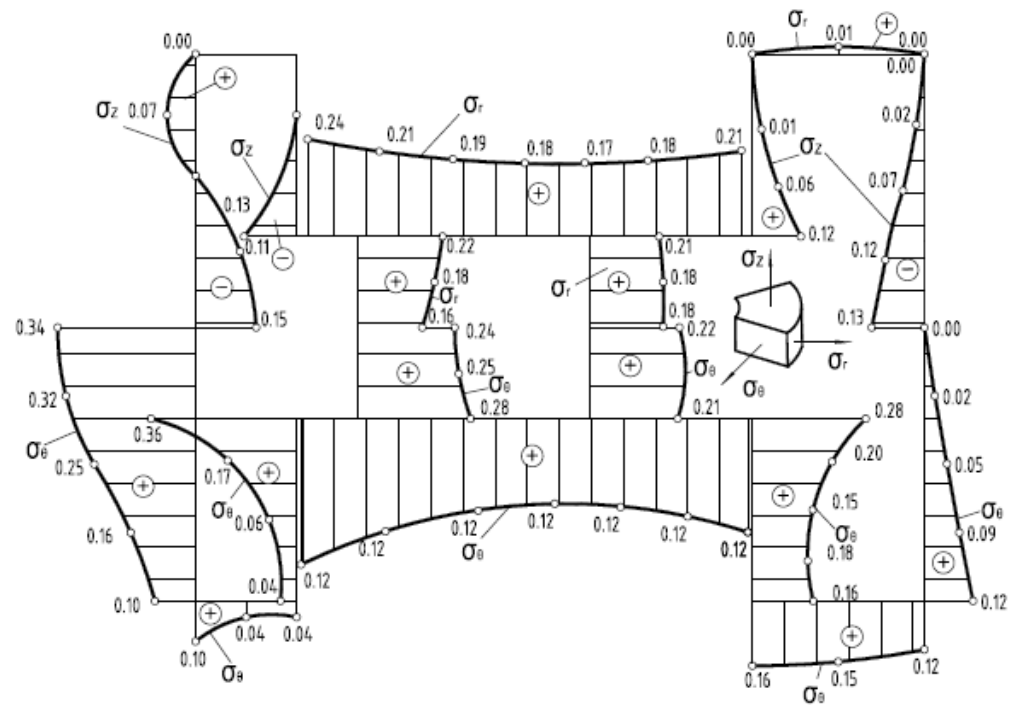

Fig. 3. Stress diagrams $\sigma_{r}, \sigma_{\theta}$ и $\sigma_{z}[\mathrm{MPa}]$ in the model of the $\mathrm{V}_{1}$ domain. 


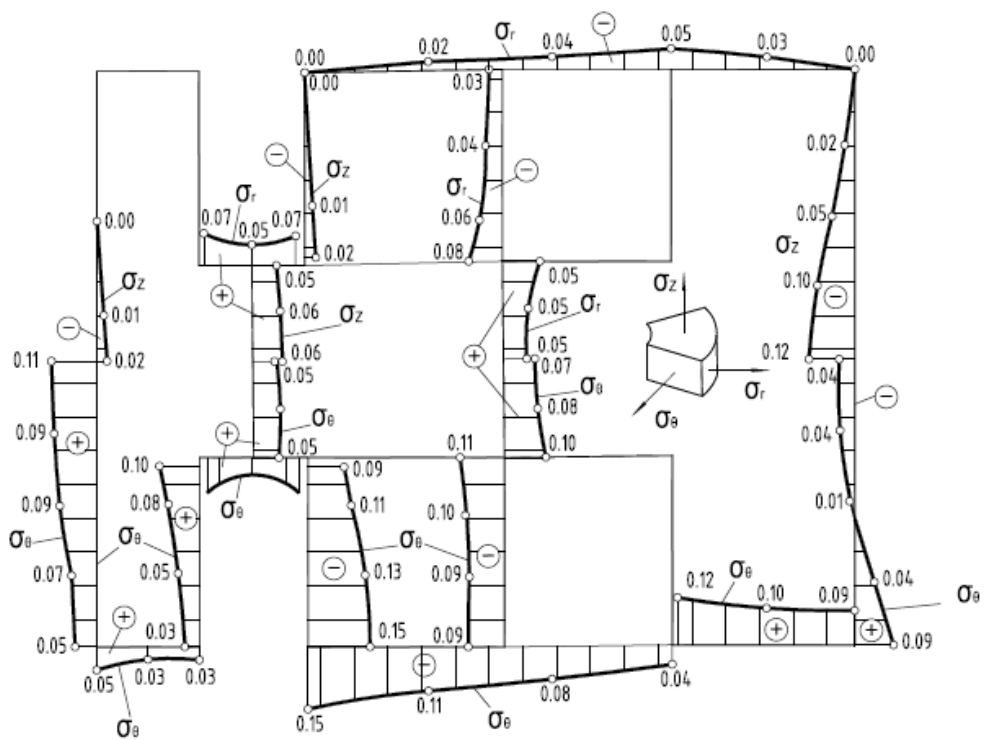

Fig. 4. Stress diagrams $\sigma_{r}, \sigma_{\theta}$ и $\sigma_{z}[\mathrm{MPa}]$ in the model, composed of areas $\mathrm{V}_{1}$ and $\mathrm{V}_{2}$, after annealing.

In accordance with the parameters of modeling of a volume static elastic problem, we obtain the following formula of transition from the stresses in the models to the stresses in a full-scale structural element:

$$
\sigma_{i j}^{(f s c)}=K_{\omega}^{2} K_{\rho} K_{l}\left[K \sigma_{i j}^{(m o d)}+(1-K)\left(\bar{\sigma}_{i j}^{(\text {mod })}-\bar{\sigma}_{i j}^{(m o d)}\right)\right],
$$

where: $K_{\omega}=\frac{\omega_{f s c}}{\omega_{\text {mod }}}, \quad K_{\rho}=\frac{\rho_{1}}{\rho_{\text {mod }}}, \quad K_{l}=\frac{l_{f s c}}{l_{\text {mod }}}, \quad K=\frac{\rho_{2}}{\rho_{1}}$;

$\sigma_{i j}^{(\text {mod })}$ - stress in the model of area $\mathrm{V}$;

$\bar{\sigma}_{i j}^{(m o d)}$ - stress in the model of area $\mathrm{V}_{1}$;

$=(\bmod )$

$\sigma_{i j} \quad$ - stresses in models made up of areas $\mathrm{V}_{1}$ and $\mathrm{V}_{2}$ after annealing.

The presented experimental studies allow us to analyze the influence of the ratio of the densities of materials from which the body is composed on the stressed state of the whole structural element.

The results of the analysis are shown in Figures 5 and 6.

For the structural element under consideration, the studies showed:

- the stresses $\sigma_{z}$ are negligible as compared with $\sigma_{r}$ and $\sigma_{\theta}$;

- the ratio of densities in a compound body effects (both quantitatively and qualitatively) on the stressed state of the structure.

Therefore, changing the ratio of densities of the materials in a compound body, i.e. choosing the materials with different volume masses, we can change the value and the distribution of stresses at either cross-section of the structural element, which is of great practical interest. 

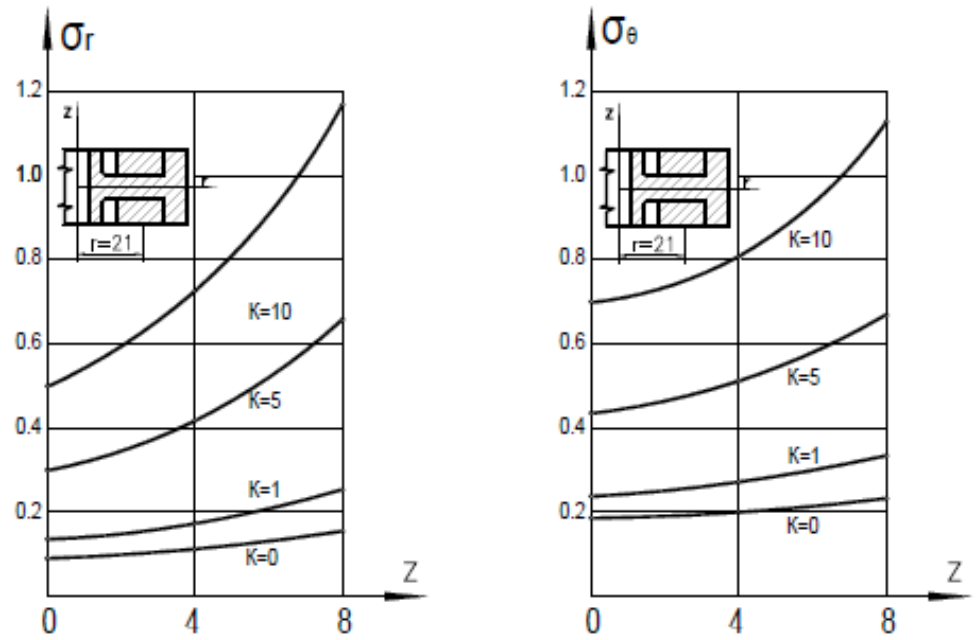

Fig. 5. The effect of the parameter $K=\rho_{2} / \rho_{1}$ on the stresses $\sigma_{r}$ and $\sigma_{\theta}[\mathrm{MPa}]$ in the crosssection $r=21$ in the fractions of $K_{\omega}^{2} K_{\rho} K_{\ell}$.
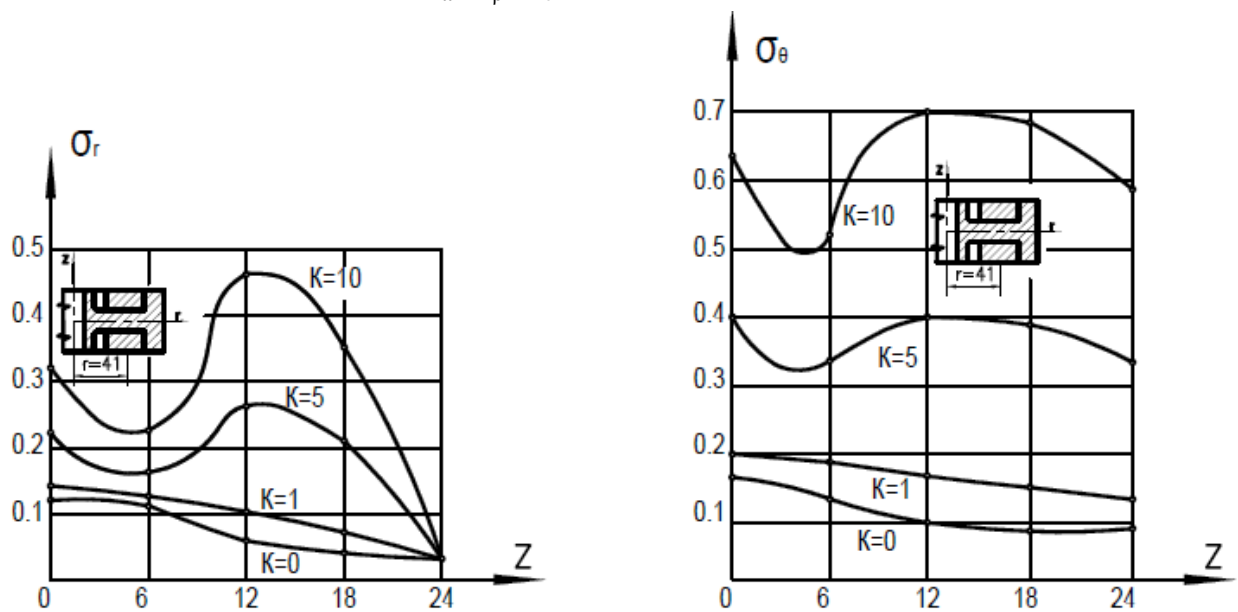

Fig. 6. The effect of the parameter $K=\rho_{2} / \rho_{1}$ on the stresses $\sigma_{r}$ and $\sigma_{\theta}[\mathrm{MPa}]$ in the crosssection $r=41$ in the fractions of $K_{\omega}^{2} K_{\rho} K_{\ell}$.

Suppose that in the considered compound structural element it is required that there were no radial expanding stresses at the outline points located at the joint of two materials. The radial expanding stresses may be eliminated through an appropriate choice of the ratio $\mathrm{K}$ of densities.

Supposing in (8) $\sigma_{r}^{(f s c)}=0$, we obtain the equation

$$
K_{\omega}^{2} K \rho K_{l}\left[K \sigma_{r}^{(m o d)}+(1-K)\left(\bar{\sigma}_{r}^{(\bmod )}-\bar{\sigma}_{r}^{=(\bmod )}\right)\right]=0,
$$

from which with known experimental values $\sigma_{r}^{(\bmod )}, \bar{\sigma}_{r}^{(\bmod )}, \bar{\sigma}_{r}^{(\bmod )}$ and $K_{\omega}^{2} K \rho K_{l} \neq 0$ we can find

$$
0,4 \mathrm{~K}+0,5(1-\mathrm{K})=0
$$




$$
\mathrm{K}=5
$$

Thus, with $K \geq 5$ there will be no radial expanding stresses at the outline points located at the joint of two materials.

\section{Conclusions}

Since the changes in the ratio of densities of materials in a compound body cause considerable changes in both the values of stresses and their distribution, it is necessary to consider the inequality of volume masses of the compound body when studying such structural elements.

\section{References}

1. G. S. Vardanyan, Applied mechanics: the use of the similarity theory methods and the methods of the analysis of dimensions for the simulation of problems in mechanics of deformable rigid bodies. Textbook, - Moscow: NITs INFRA-M, 2016, -174 p.

2. E. K. Agakhanov, M. K. Agakhanov, About the possibility of the use of the effect equivalence in analytical solutions to the elasticity theory problems // MGSU Bulletin, 4/2010, No. 3, p.p. 140-143.

3. E. K. Agakhanov, M. K. Agakhanov, About the simulation of the space-forces effect in a creep elastic body // Higher education proceedings. North-Caucasus Region. Engineering sciences. -2005. No. 1, p. 25.

4. E. K. Agakhanov, M. K. Agakhanov, Modeling of weighing and filtration forces in the “dam-foundation" system / MATEC Web Conf. 86, 01012 (2016)

5. B. N. Ushakov, I. P. Frolov, Stresses in composite structural elements. Moscow, Mashinostroyenie, 1979, $134 \mathrm{p}$.

6. B. N. Ushakov, Study of stresses in composite structural elements. VIII All-Union conference devoted to the photo-elasticity method. Tallinn, 1979, p.p. 283-290.

7. Z. E. Abdulaliev, Determination of temperature stresses in multi-material workpieces. Plant laboratory, 1970, No. 3, p.p. 347-349.

8. A. K. Preiss, F. A. Granenko, Application of the "freezing" method to the determination of stresses on rotating models. The polarization-optics method of study of stresses, edited by Prigorovsky N. I., Moscow, 1956, p.p. 271-279. 